\title{
LETTERS
}

The purpose of this Letters section is to provide rapid dissemination of important new results in the fields regularly covered by Physics of Fluids. Results of extended research should not be presented as a series of letters in place of comprehensive articles. Letters cannot exceed four printed pages in length, including space allowed for title, figures, tables, references and an abstract limited to about 100 words. Ordinarily, there is a three-month time limit, from date of receipt to acceptance, for processing Letter manuscripts. Authors must also submit a brief statement justifying rapid publication in the Letters section.

\section{Vortex ring pinchoff in the presence of simultaneously initiated uniform background co-flow}

\author{
Paul S. Krueger \\ Department of Mechanical Engineering, Southern Methodist University, Dallas, Texas 75275

\begin{abstract}
John O. Dabiri and Morteza Gharib
Graduate Aeronautical Laboratories, California Institute of Technology (GALCIT), Pasadena, California 91125
\end{abstract}

(Received 16 January 2003; accepted 25 April 2003; published 6 June 2003)

\begin{abstract}
Vortex rings were formed with a piston-cylinder mechanism in the presence of uniform background co-flow supplied through a shroud surrounding the cylinder. The jet and co-flow were started simultaneously. Ratios of the co-flow to jet velocity $\left(R_{v}\right)$ in the range $0-1$ were considered. The formation number $(F)$ as a function of $R_{v}$ was determined using the procedure of Gharib et al. [J. Fluid Mech. 360, 121 (1998)] and a generalized definition of formation time. The results show a sharp decrease in $F$ as $R_{v}$ increases from $0.5-0.75$, suggesting possible performance limitations for pulsed-jet propulsion. (C) 2003 American Institute of Physics. [DOI: 10.1063/1.1584436]
\end{abstract}

The investigation of vortex ring formation in a pistoncylinder mechanism by Gharib et al. ${ }^{1}$ demonstrated the existence of a non-dimensional time scale (formation number, $F$ ) at which the circulation in the forming vortex ring saturates and the ring separates, or pinches off, from the generating jet in terms of its entrainment of vorticity. After pinchoff, the remainder of the generating jet follows the vortex ring as a trailing jet. The practical significance of this result has been recently demonstrated by Krueger and Gharib ${ }^{2}$ who showed that the average thrust during a pulse is maximized by pulses of non-dimensional duration very near the formation number. Hence, the formation number has direct relevance to applications where pulsed jets are used to impart momentum to the flow, such as flow control or pulsed-jet propulsion. Such applications typically involve the ejection of pulses into background flow [cross flow in the case of flow control or parallel flow (co-flow) in the case of propulsion]. Gharib et al. ${ }^{1}$ and Krueger and Gharib, ${ }^{2}$ on the other hand, considered only quiescent ambient flow. It is therefore relevant to consider the effect of background flow on the formation number. Motivated by the application to propulsion, this investigation considers the effect of uniform background coflow on the formation number of vortex rings formed by a piston-cylinder mechanism when the co-flow and jet flow are started simultaneously. Simultaneous initiation of the flows is most relevant for jet propulsion devices accelerated from rest or nearly from rest.

Vortex ring formation and pinchoff was studied experimentally using a piston-cylinder vortex ring generator in water with a piston diameter of $D=2.54 \mathrm{~cm}$. The co-flow was supplied through a clear, Plexiglas shroud surrounding the vortex ring generator, ${ }^{3}$ as shown in Fig. 1. A constant-head tank supplied flow to the vortex ring generator while an independent pump supplied the co-flow. Separate solenoid valves, actuated by a computer, controlled the initiation of each flow, allowing independent actuation of the jet and coflow velocities. The flow rates were measured using Transonic Systems T-110 flow rate sensors, providing measurements of the time-varying piston and co-flow velocities, $U_{p}(t)$ and $V_{c}(t)$, respectively.

Digital particle image velocimetry (DPIV) was used to measure the velocity field and azimuthal vorticity, $\omega_{\theta}$, in the region $\{0 \leqslant x / D \leqslant 5.0,0 \leqslant r / D \leqslant 2.4\}$. For these measurements, the flow was seeded with $20 \mu \mathrm{m}$, neutrally buoyant, silver coated, hollow glass spheres. The particles were illuminated with a pulsed Nd:YAG laser and imaged through the shroud with a UNIQ Vision UP-1830 CCD camera at $30 \mathrm{fps}$. The particle images were processed with an in-house code employing a window-shifting algorithm for improved accuracy. The spacing of the vectors in the processed data was $0.08 D \times 0.08 D$.

Using this apparatus, vortex ring formation is observed under the conditions where the jet and co-flow velocities are started simultaneously and allowed to ramp up to steadystate values $U_{0}$ and $V_{0}$, respectively. A sample case is shown in Fig. 2 for a velocity ratio of $R_{v}=0.50$ and a Reynolds number of $\operatorname{Re}_{s}=1300$ where

$$
R_{v} \equiv \frac{V_{0}}{U_{0}}
$$




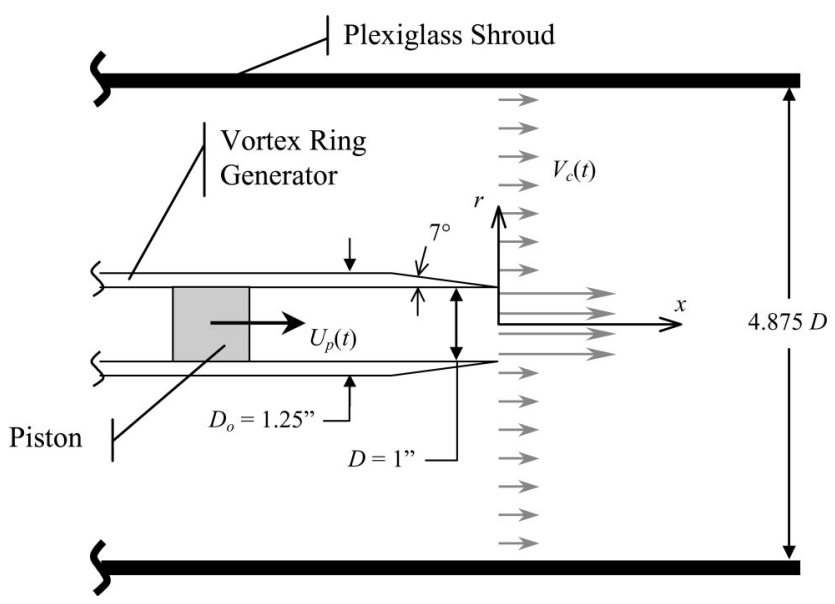

FIG. 1. Cross section of the experimental setup near the nozzle exit plane.

$$
\operatorname{Re}_{s} \equiv \frac{\left|U_{0}-V_{0}\right| D}{\nu}=\operatorname{Re}_{j}\left|1-R_{v}\right|
$$

$\operatorname{Re}_{s}$ is the Reynolds number based on the shear layer strength (at steady state) and $\operatorname{Re}_{j}$ is the steady state jet Reynolds number, namely $U_{0} D / \nu$. Runs were conducted at $R_{v}=0$, 0.25 , and 0.50 for $U_{0}=11.4 \mathrm{~cm} / \mathrm{s} \quad\left(\operatorname{Re}_{s}=2600,1940\right.$, and 1300, respectively), and at $R_{v}=0,0.50,0.75,1.0$ for $U_{0}$ $=5.5 \mathrm{~cm} / \mathrm{s} \quad\left(\operatorname{Re}_{s}=1250,624,312 \text {, and } 0 \text {, respectively }\right)^{4}$ (Note that $R_{v}=0$ corresponds to the case of no co-flow as studied by Gharib et al. ${ }^{1}$ ) The ramp-up time for the jet velocity was $0.19 \pm 0.02 \mathrm{~s}$ for the $U_{0}=11.4 \mathrm{~cm} / \mathrm{s}$ cases and $0.12 \pm 0.02 \mathrm{~s}$ for the $U_{0}=5.5 \mathrm{~cm} / \mathrm{s}$ cases. The ramp-up time for the co-flow velocity varied between 0.2 and $0.3 \mathrm{~s}$ (shorter time for lower $V_{0}$ ). The jet and co-flow were sustained long enough to observe pinchoff, so the shutdown characteristics of the flows were not relevant to the investigation. The timevarying total circulation, $\Gamma$, was determined from DPIV measurements of the vorticity as

$$
\Gamma=\int \omega_{\theta} d r d x
$$

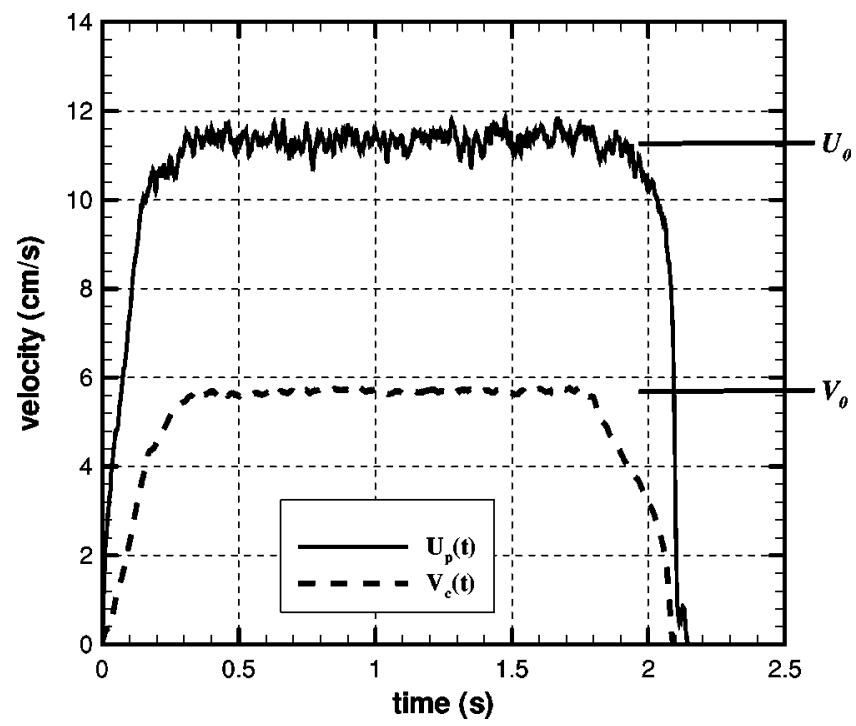

FIG. 2. Piston and co-flow velocity traces for $R_{v}=0.50, U_{0}=11.4 \mathrm{~cm} / \mathrm{s}$. The velocities near shut down $(t>1.5 \mathrm{~s})$ are not relevant to this study. where the integration is over the vorticity in front of the nozzle.

Using the DPIV measurements of $\Gamma$ and following Gharib et al., ${ }^{1}$ the formation number, $F$, of the vortex rings studied here is defined as the formation time at which the total circulation is equal to the circulation in the pinched off vortex ring (see, for example, Fig. 7 in Gharib et al. ${ }^{1}$ ). At this point it is relevant to consider how formation time should be defined when co-flow is included in the formation process. Gharib et al. ${ }^{1}$ defined formation time as

$$
\hat{t}_{G R S}=\frac{t \bar{U}_{p}(t)}{D}=\frac{X_{p}(t)}{D}
$$

where $\bar{U}_{p}(t)$ is the running average of the piston velocity and $X_{p}(t)$ is the piston displacement as a function of time [i.e., $X_{p}(t) / D$ is the piston stroke-to-diameter ratio]. For the case with co-flow, we generalize the definition of formation time to

$$
\hat{t}=\frac{t\left(\bar{U}_{p}(t)+\bar{V}_{c}(t)\right)}{D},
$$

where $\bar{V}_{c}(t)$ is the running average of the co-flow velocity. Note that $\hat{t}=\hat{t}_{G R S}$ when $V_{c}(t)=0$.

The motivation for the definition of formation time in Eq. (5) is based on the slug model. Although the slug model consistently underestimates vortex ring circulation, especially for small stroke ratios, it does provide a good approximation of $\partial \Gamma / \partial t$ for stroke ratios greater than $1 .^{5}$ Since vortex ring pinchoff typically occurs for stroke ratios greater than 1 , the slug model should serve as at least an approximate guide for determining the scaling relevant to measurements of formation number. With this caveat in mind, the slug model predicts

$$
\frac{\partial \Gamma}{\partial t}=\int_{0}^{\infty}-u \frac{\partial u}{\partial r} d r=-\frac{1}{2} \int_{r=0}^{r=\infty} d\left(u^{2}\right),
$$

where $u$ is the axial component of velocity. For the case of an impulsively started jet and co-flow [i.e., $U_{p}(t)=U_{0}$ and $V_{c}(t)=V_{0}$ for $\left.t>0\right]$, Eq. (6) reduces to

$$
\frac{\partial \Gamma}{\partial t}=\frac{1}{2}\left(U_{0}^{2}-V_{0}^{2}\right),
$$

where the jet centerline velocity ( $u$ at $r=0$ ) is approximated by the piston velocity. For the special case of an impulsively started jet with no co-flow $\left(V_{0}=0\right)$, substituting $\hat{t}_{G R S}$ into Eq. (7) gives

$$
\frac{\partial \hat{\Gamma}_{R}}{\partial \hat{t}_{G R S}}=\frac{1}{2}
$$

where

$$
\hat{\Gamma}_{R}=\frac{\Gamma}{U_{0} D},
$$

is the scaling of circulation adopted by Rosenfeld et al. ${ }^{6}$ If impulsively started co-flow is included, the obvious scaling of $\Gamma$ is 


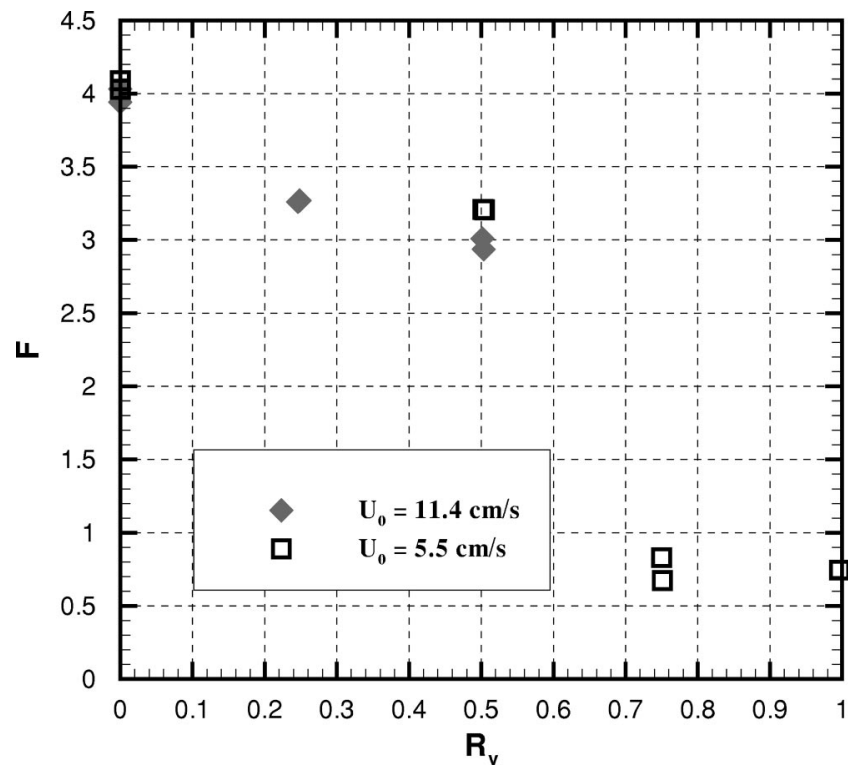

FIG. 3. Formation number as a function of velocity ratio.

$$
\hat{\Gamma}=\frac{\Gamma}{\left(U_{0}-V_{0}\right) D},
$$

since the strength of the vortex sheet supplied by the jet shear layer scales with $\left(U_{0}-V_{0}\right)$. Using Eq. (10) and the generalized definition of formation time [Eq. (5)], Eq. (7) reduces to $\partial \hat{\Gamma} / \partial \hat{t}=1 / 2$. Thus, the proposed generalization of formation time when co-flow is present preserves the scaling of $\partial \Gamma / \partial t$ for the special case of impulsively started flow, independent of the co-flow magnitude, $V_{0}$. The preservation of the scaling of $\partial \Gamma / \partial t$ for the case of impulsively started flow combined with the fact that $\hat{t}=\hat{t}_{G R S}$ when $V_{c}(t)=0$ leads the authors to believe that Eq. (5) gives the proper generalization of formation time when co-flow is present.

Using Eq. (5) as the definition of formation time and the general definition of the formation number given previously, the formation number, $F$, is the $\hat{t}$ at which the circulation in the pinched off vortex ring has been ejected. That is, circulation ejected when $\hat{t}>F$ is not entrained by the leading vortex ring. For simultaneous initiation of the jet and coflow, $F$ should be a function of $R_{v}, \operatorname{Re}_{s}=\operatorname{Re}_{j}\left|1-R_{v}\right|$, and the shape of the velocity profiles during the initiation or "rampup" period. Since the velocity profiles are approximately the same for all cases considered and $\mathrm{Re}_{s}$ is high enough (for $R_{v} \leqslant 0.75$ ) that the formation process is primarily inviscid, $F$ is expected to be primarily a function of $R_{v}$ for the cases considered.

Measurements of the formation number as a function of $R_{v}$ are shown in Fig. 3 for $U_{0}=11.4 \mathrm{~cm} / \mathrm{s}$ and $5.5 \mathrm{~cm} / \mathrm{s}$. Multiple points at the same $R_{v}$ indicate multiple trials at the same conditions. A key component for these measurements is the comparison of the total circulation ejected as a function of $\hat{t}$ with the circulation of the pinched off vortex ring. The total circulation measurements were obtained from the DPIV measurements of vorticity as described previously. The ring circulation was measured by integrating the vorticity within an isovorticity contour of magnitude $2 \mathrm{~s}^{-1}$ surrounding the
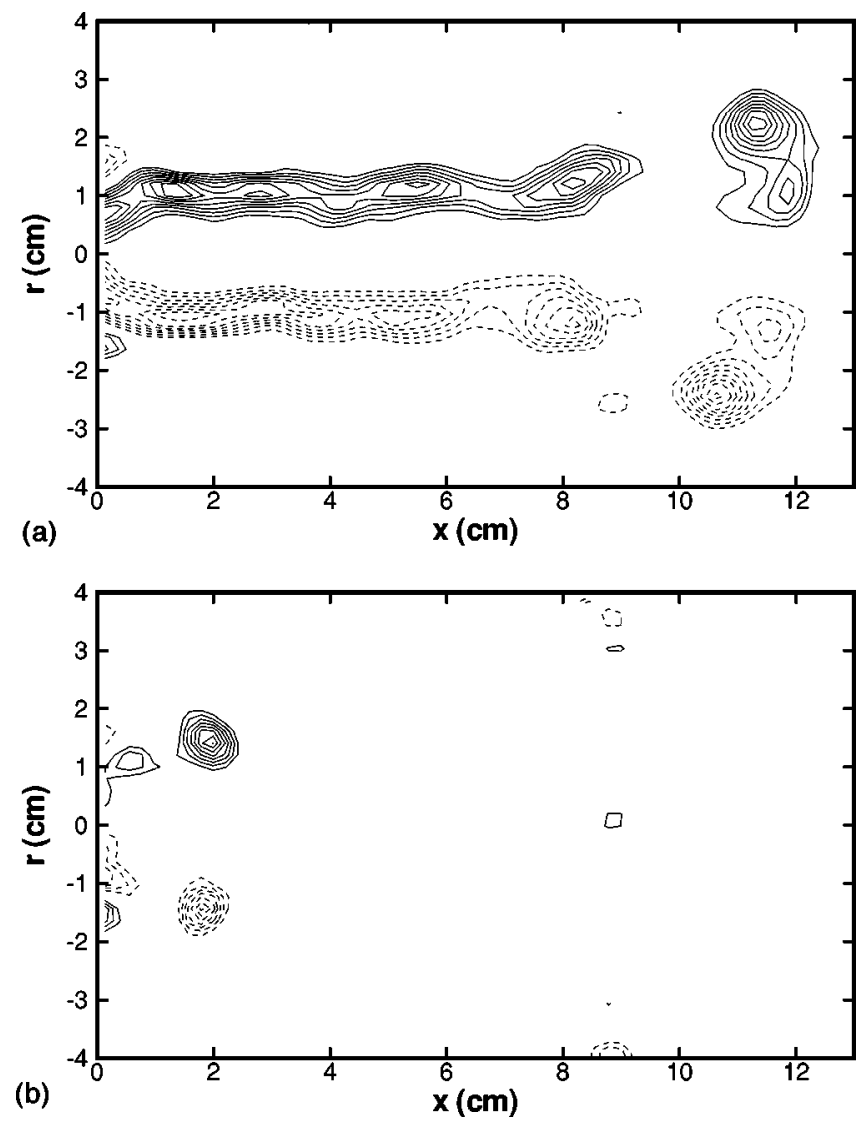

FIG. 4. Vorticity plots at instants just after pinchoff is completed for (a) $R_{v}=0.50$ and (b) $R_{v}=0.75$, both at $U_{0}=5.5 \mathrm{~cm} / \mathrm{s}$. The dashed contours represent negative vorticity. The minimum contour plotted of a given sense is $2 \mathrm{~s}^{-1}$.

ring, after this contour was observed to separate from the vorticity of the generating jet (i.e., after the leading vortex ring had pinched off, as illustrated for two cases in Fig. 4). Comparing the ring circulation (which remains nearly constant after pinchoff) with the total circulation (which increases with $\hat{t}$ ) determines the $\hat{t}$ at which the total and ring circulations are equal, and hence, the formation number. This follows the procedure used by Gharib et al. ${ }^{1}$ The uncertainty in the measurements of formation number attained this way is reflected by the spread in the data points where multiple trials were performed. ${ }^{7}$

Figure 3 shows good agreement between the results for $U_{0}=11.4 \mathrm{~cm} / \mathrm{s}$ (filled symbols) and $U_{0}=5.5 \mathrm{~cm} / \mathrm{s}$ (open symbols) at $R_{v}=0$ and 0.50 , confirming that $F$ is nearly independent of $\mathrm{Re}_{s}$ for the cases considered. The formation number at $R_{v}=0$ is approximately 4 , in agreement with the no co-flow measurements of Gharib et al. ${ }^{1}$ As $R_{v}$ increases from 0 to $0.5, F$ decreases from 4 to about 3 . This trend is not surprising since increasing $R_{v}$ decreases the strength of the shear layer feeding the ring and increases the rate at which the ring is advected away from the nozzle, both of which impede the growth of the leading vortex ring. Between $R_{v}=0.5$ and 0.75 , however, there is an abrupt drop in the formation number from approximately 3 to less than 1 . At $R_{v}=1.0$, the formation number is not zero (a distinct, but small, vortex ring is formed) despite the fact that the vorticity flux is zero once the jet and co-flow have reached their 
steady-state values. ${ }^{8}$ This is due to a slightly faster ramp-up of the jet velocity relative to the co-flow velocity during flow initiation.

While an overall decrease in $F$ with $R_{v}$ is to be expected (under ideal conditions $F$ would be zero at $R_{v}=1$ ), the dramatic change in $F$ between $R_{v}=0.5$ and 0.75 was not anticipated, especially since the vorticity flux for $R_{v}=0.75$ is still quite substantial. Indeed, comparison of the DPIV measurements for the $R_{v}=0.75$ and $R_{v}=1.0$ cases shows them to be remarkably similar, indicating that the formation process of the vortex ring is preempted very near flow initiation at $R_{v}$ $=0.75$. In contrast, the vortex ring develops smoothly and pinchoff is observed further downstream for the cases with $R_{v} \leqslant 0.5$. The sharp distinction between ring formation for $R_{v} \leqslant 0.5$ and $R_{v}=0.75$ is illustrated in Fig. 4, which shows vorticity contour plots for $R_{v}=0.5$ and 0.75 with $U_{0}$ $=5.5 \mathrm{~cm} / \mathrm{s}$ at instants just after the completion of the pinchoff process (note the disconnection between the ring and jet vorticity). The dramatic difference between $R_{v}=0.5$ and 0.75 can be seen not only in the reduced strength of the pinched off vortex ring for $R_{v}=0.75$, but also in the relatively quick completion of the pinchoff process at $R_{v}=0.75$ as indicated by the proximity of the pinched off ring to the nozzle ( $x$ $=0)$ in Fig. 4(b).

Given the importance of vortex ring formation for the impulse supplied by starting jets (Krueger and Gharib ${ }^{2}$ ), the dramatic change in the character of vortex ring formation in the presence of co-flow observed near $R_{v}=0.5$ could have significant implications for the performance of pulsed-jet devices or unsteady jet propulsion in the presence of background flow. This is not to say that the observed trend with $R_{v}$ is "universal" in the sense that a sudden decrease in formation number should always be expected in the range
$0.5<R_{v}<0.75$. On the contrary, the fact that vortex ring formation seems to halt very near flow initiation for the $R_{v}$ $=0.75$ case $[$ see Fig. 4(b)] suggests changes in the manner of flow initiation may significantly affect the observed transition in formation number. Nevertheless, the mere existence of such a dramatic drop in the formation number could have important ramifications for pulsed-jet devices.

The authors are currently working to obtain measurements of the formation number at more points in the range $0.5<R_{v}<0.75$ to better resolve this transition and develop a physical explanation of the phenomenon. This continued work will be the subject of a later manuscript.

\footnotetext{
${ }^{1}$ M. Gharib, E. Rambod, and K. Shariff, "A universal time scale for vortex ring formation," J. Fluid Mech. 360, 121 (1998).

${ }^{2}$ P. S. Krueger and M. Gharib, "The significance of vortex ring formation to the impulse and thrust of a starting jet," Phys. Fluids 15, 1271 (2003).

${ }^{3}$ Flow visualization of ring formation and measurements of ring velocity with and without the shroud in place confirmed that the shroud had negligible effect on vortex ring formation.

${ }^{4}$ Due to limitations of the pump supplying the co-flow, $R_{v}>0.50$ could not be achieved for $U_{0}=11.4 \mathrm{~cm} / \mathrm{s}$.

${ }^{5} \mathrm{~N}$. Didden, "On the formation of vortex rings: Rolling-up and production of circulation," Z. Angew. Math. Phys. 30, 101 (1979).

${ }^{6}$ M. Rosenfeld, E. Rambod, and M. Gharib, "Circulation and formation number of laminar vortex rings," J. Fluid Mech. 376, 297 (1998).

${ }^{7}$ At $R_{v}=0.5$, additional uncertainty is present because the pinchoff process is completed near the end of the shroud where the co-flow obtains a slight radial component. This may affect the pinchoff process, but the authors estimate the uncertainty in the reported formation numbers at $R_{v}=0.5$ to be within \pm 0.5 .

${ }^{8}$ For the case where $R_{v}=1$, the finite thickness of the vortex ring generator and the relatively large $\operatorname{Re}_{j}\left(\operatorname{Re}_{j}=2600\right.$ for $U_{0}=11.4 \mathrm{~cm} / \mathrm{s}$ and $\operatorname{Re}_{j}$ $=1250$ for $U_{0}=5.5 \mathrm{~cm} / \mathrm{s}$ ) leads to the formation of an annular wake behind the vortex ring generator after the jet and co-flow reach steady-state values. The vorticity in this wake decreases to zero as the co-flow and jet mix downstream of the nozzle exit.
} 